Results and discussion In the control group (not receiving a pharmacological correction) that underwent local cooling and withdrawn from the experiment immediately after the cold exposure, the following morphological changes are observed in the pre-active period of the cold trauma: the boundaries between the layers of the epidermis and the cells themselves are stilted, the nuclei of the cells of the basal and granular layers are picotized, .e. phenomena of paranecrosis are observed. The dye stains the epidermis cells diffusely. The cytoplasm of epithelial cells is vacuolized. From the side of the vessels of the papillary layer of the dermis, stasis phenomena are observed, a persistent spasm of the dermis vessels is noted, which indicates the cold stress. Erythrocytes are glued together in "coin pillars" and capillaries are clogged, and the phenomena of occlusion of the microcirculatory system are visible.

With topical application of dihydroquercetin from the side of the epidermis, the peeling effect is observed, which indicates the keratolytic action of the dihydroquercetin preparation, the keratinization processes proceed without any peculiarities. From the side of the dermis, the edema of the middle degree of expression in the reticular layer is revealed, in addition, there is an increase in the fibroblastic reaction. Thus, one can judge the positive effect of the drug on water-electrolyte, protein metabolism in the skin and stimulating effect on the synthesis of glycosaminoglycans with topical application of dihydroquercetin. In the subgroup subjected to local cooling, against the backdrop of topical application of dihydroquercetin, and the inferior release from the experiment, compared with the control subgroup, less pronounced phenomena of paranecrosis are noted. From the side of the vessels of the papillary layer of the dermis, the phenomenon of stasis is manifested to a lesser degree, which indicates the cryoprotective action of dihydroquercetin in topical application.

With oral administration of dihydroquercetin in a subgroup subjected to local cooling, the phenomena of paranecrosis are less than in the control group. However, the cryoprotective effect for oral administration is less pronounced than with local use of the preparation, which indicates a rapid biotransformation of the drug for oral administration.

Thus, against the background of the use of dihydroquercetin, a visible cryoprotective effect is observed, which is most pronounced when applied locally, which indicates a rapid biotransformation of flavonoids by oral administration.

References:

1. Kotelnikov V.P. Frostbites. - M .: Medicine, 1988. -256c.

2. Myader OD, Adaskevich VP Morphofunctional dermatology - Moscow 2006 - P. 655-656.

3. Dorovskih V.A., Borodin E.A., Tseluyko S.S., The antioxidants in the prevention and korrektsii cold stress. - Blagoveshchensk: AGMA - 2001. -183 p.

4. Myadelets O.D., Adaskevich V.P., Morfofunktcionalnaja dermatology - Moscow 2006 - P. 655-656.

5. $\quad$ Nozdrin V.I., Barashkova S.A., Semchenko V.V. Leather and its derivatives - Omsk - Eagle - 2005 - 7-24.

6. Teselkin Y.O., Zhambalova B.A., Babenkova I.V. and others. The antioxidant properties of DHQ Tutorial "Biophysics" in 1996. T. 41, no. 3, pp 620 - 624.

7. Gnaiger E., Kuznetsov A. V., Rieger O., Amberger A., Fuchs A., Stadlmann S., Eberl T., Margreiter R. Mitochondrial defects by intracellular calcium overload versus endothelial coldischaemia/reperfuston injury // Transplant International. - 2000. - T. 13. N.7. - P. 555-557.

\title{
DOI 10.22448/AMJ.2017.3.53-54 \\ CLINICAL APPLICATION STATUS ON ANTI-INFLAMMATORY OF TRADITIONAL CLASSICAL FORMULA-ZHIBAIDIHUANG PILL
}

\section{Mingjiyang1, Dingyan1, Liyang1, Guoshuang1, Jiangxicheng*}

(1. Heilongjiang university of Chinese medicine, the Harbin city of heilongjiang province 150040;)

Abstract:Objective: To provide basis and reference data for the clinical application and pharmacological research onanti-inflammatory of Zhibai dihuang pill. Methods: The data and information from related literature prove the clinical effect of Zhibai dihuang pill, including research thought, method and progress . Introducing the ideas, methods and progress of clinical effect on Zhibai dihuang pill. Results: This paper summarizes the research progress of the Zhibai dihuang pill's effect on anti-inflammatory. Conclusion: the zhibai dihuang pill could cure various diseases caused by fire excess from yin deficiency, and adjust the level of the index factors associated with disease.

keywords : the zhibai dihuang pill, Clinical application

Zhibaidihuang pill consiste of Anemarrhena, phellodendron, Radix Rehmanniae Preparata, Fructus Corni, peony bark, Chinese yam, Poria, Alisma, it could cure treatment of hyperactivity of fire due to yin deficiency, such as hot flashes sweating, dry mouth and sore throat, tinnitus, spermatorrhea, short red urine and other symptoms. zhibaidihuang pill has anti-inflammatory effect, including Senile vaginitis, periodontitis, gingivitis, nephritis and so on. It summarized as follows.

1 Senile vaginitis Zhibaidihuang Pill vaginitis has obvious effect mainly for senile on treatment of vaginitis. Senlian Ruan[1] studied the clinical effect of Zhibaidihuang pill in treatment of senile vaginitis. She studied in 120 patients with senile vaginitis, and divided them randomly into observation group and control group, which has 60 patients in each group. The Patients in the control group was treated with Metronidazole Tablets and the patients in the observation group were treated with Zhibaidihuang pill. Comparing the therapeutic effects of two groups, we find that the 
total curative effect of the observation group was significantly higher than that of the control group, and the incidence of adverse reactions in the observation group was also less than that in the control group. Its indicate that the treatment has a significant effect of Zhibai Dihuang Pill on senile vaginitis.

2 periodontitis and gingivitis Luo Xia [2] [3] respectively with randomly selected chronic periodontitis patients as the research object, divided into two groups, the control group with routine periodontal treatment, the experimental group used zhibodihuang treatment, analysis of two groups of treatment effect. two groups is significant difference $(\mathrm{P}<0.05)$ after treatment, compared with of periodontal indexes, IL-6, IL-1, TNF- $\alpha, C$ reactive protein and total clinical efficiency, The experimental results show zhibaidihuang pill combined with periodontal therapy has good curative effect on redution chronic periodontitis inflammatory factors, not only enhance the treatment effect, effectively reduce the inflammatory factor can promote the repair and regeneration of periodontal tissues. Huang Jianyu [4] selected recurrent episodes of acute gingivitis patients as the research object, observated the inflammation level before and after treatment. The results show that Zhibaidihuang pill combined with antibiotics can treat acute gingivitis.

3 nephritis Zhibaidihuang decoction treat the patients with acute pyelonephritis, the control group was given antibiotic treatment, the treatment group was given oral eight powder and Zhibaidihuang pill . The positive incidence of urine bacteria culture, the main symptoms and signs disappeared time of patients in the treatment group were better than those in the control group [5], yung-Ho Hsu[6] proved that zhibaidihuangwan has a protective effect on apoptosis of rat kidney cells, which happened by gentamicin

4 male reproductive inflammatory diseases Donghua [7] reviewed the clinical situation, the research progress and the treatment mechanism about object for each type of male infertility,. Liu Yongxin [8] reported Zhibaidihuang decoction combined with massage therapy has significant curative effect for spermatorrhea.

Wu Jinping [9] proved that Zhibaidihuang pill combined erzhi pill has good curative effect on chronic Vesiculitis. The control group was given roxithromycin + Pudilanxiaoyan tablets treatment; the observation group was given with Zhibaidihuang pill combined erzhi pill treatment, the observation group cure time was significantly shorter than the control group, and semen volume and sperm motility index in observe group increased more significantly than the control group.

In conclusion, Zhibaidihuang pill have curative effect on anti-inflammatory effect. In the study, the research methods was devolopment from the treatment rate of simple summary expanded to statistical analysis, the index from the macro effect of in-depth observation into the cell or the microscopic factor, it is provide basis for clinical application and modern pharmacological study of Zhibaidihuang pill.

\title{
Reference
}

1 Ruan Senlian, Asia, Meng Xiangling. Zhibaidihuang pill in the treatment of senile vaginitis clinical curative effect analysis of // and clinical pharmacology of traditional Chinese medicine. - 2015 (01) - C.302

2 Luo Lihong. Zhibaidihuang pill for chronic periodontal maintenance therapy the clinical curative effect of // in northern medicine- $2016(04)$ - C. 82-83.

3 Xia. Chengjian Zhibaidihuang Decoction on chronic periodontitis inflammatory factors // and clinical pharmacology of traditional Chinese medicine-2015 (01) - C.277-278.

4 Huang Jianyu. Zhibaidihuang pill in the treatment of 12 cases of acute gingivitis // Guangdong occupation technology education and research- 2016 (04) - C.194-196.

5 Yang Lin, Wang Rong. Eight is scattered and Zhibaidihuang pill in the treatment of 60 cases of acute pyelonephritis with // Chinese TCM modern distance education-2014 (09) - C.132-133.

6 Yung-HoHsu, Tso-Hsiao Chen, Mei-Yi Wu, et al. Protective effects of Zhibai Dihuang Wan on renal tubular cells affected with gentamicin-induced apoptosis // Journal of Ethnopharmacology. -2014-151(2014) - C.635-642.

7 Han Zhong, Donghua, where the application of Qing Hu zhibaidihuangwan in male infertility //.China science, -2016 (12) C.84-87.

8 Liu Yongxin, Yang Chunmei. Zhibaidihuang decoction combined with massage therapy efficacy // Chinese spermatorrhea community physicians-2016(17) - C.101+103.

9 Wu Jinping, Zheng Jianlong, Wei Mingquan, Liu Yannan. Two to zhibaidihuangwan combined pill in the treatment of chronic Vesiculitis curative effect observation // new Chinese medicine- 2015(12) - C.84-86.

\section{DOI 10.22448/AMJ.2017.3.54-55 \\ CLINICAL OBSERVATION ON THE TREATMENT OF MILD COGNITIVE IMPAIRMENT BY COMBINED ACUPUNCTURE AND MEDICATION}

\author{
PangJing \\ The Second Affiliated Hospital of Heilongjiang TCM University, 150001, Harbin, China ; EMAIL : XKP850713@126. \\ COM
}

Abstract : To observe the therapeutic effect of acupuncture plus medication in the treatment of mild cognitive impairment of liver-kidney insufficiency type.

Key words:Acupuncture;Acupuncture medication combined;Mild cognitive impairment; donepezil

54 Амурский медицинский журнал №3 (19) 2017 